\title{
DNA methylation is associated with the lipoprotein profile in an elderly population
}

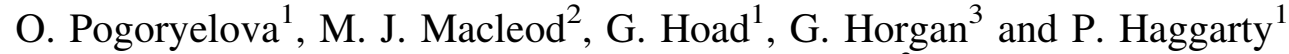 \\ ${ }^{1}$ Rowett Institute of Nutrition \& Health, University of Aberdeen, Aberdeen, UK, ${ }^{2}$ University of Aberdeen, Division of Applied \\ Medicine, Aberdeen, UK and ${ }^{3}$ BiOSS Rowett Institute of Nutrition and Health at the University of Aberdeen
}

\begin{abstract}
Atherosclerosis and dyslipidaeamia are major factors predisposing to cardiovascular disease and stroke. Diet and lifestyle are known to influence the risk of cardiovascular disease but the mechanisms linking such risk factors to disease are not fully understood. Epigenetic mechanisms, such as DNA methylation, regulate tissue specific gene expression, they are responsive to lifestyle and diet ${ }^{(1)}$ and have been implicated in a number of disease states.

We hypothesized that DNA methylation in selected genes is associated with the blood lipoprotein profile in an elderly population. The study population consisted of Aberdeen birth cohort (ABC) 1921 and 1936. Volunteers born in Aberdeen in 1921 and 1936 were recruited and blood samples were collected in 1997 as a part of Scottish Mental Survey ${ }^{(2)}$. At the time of sample collection they were 77 and 62 years old respectively. DNA methylation status was measured in two genes - IL10, and SOD3 - implicated in aging, atherosclerosis and inflammation. SOD3 plays protective role in oxidative stress and its demethylation has been observed in atherosclerotic aorta ${ }^{(3)}$. IL10 plays anti-inflammatory role and improves the resistance of tissues to insufficient blood supply or ischemia ${ }^{(4)}$. Methylation was determined by pyrosequencing (PyroMark MD Qiagen, Crawley, UK) after bisulphite conversion of lymphocyte DNA using Epitect Bisulfite kits (Qiagen, Crawley, UK).

SOD3 methylation was positively associated with total cholesterol (TC) $n=559, p<0.001, R^{2}=0.022$, high density lipoproteins (HDL) $n=559, p=0.012, R^{2}=0.011$ and low density lipoproteins (LDL) $n=555, p=0.011, R^{2}=0.012$. Anti-inflammatory $I L 10$ also showed a positive correlation with HDL $\left(n=568, p=0.012, R^{2}=0.011\right)$. For TC the associations remained significant when each of the cohorts was analysed separately.

In this association study we have demonstrated a relationship between DNA methylation of genes involved in oxidative stress (SOD3) and inflammation (IL10) and blood lipoprotein status in a large elderly population. This association study does not establish causality but it suggests that epigenetic processes, particularly in relation to cardiovascular disease and epigenetic control of SOD3 and IL10, merit further study.
\end{abstract}

1. Scoccianti C, Ricceri F, Ferrari P, Cuenin C, Sacerdote C, Polidoro S, et al. Methylation patterns in sentinel genes in peripheral blood cells of heavy smokers: Influence of cruciferous vegetables in an intervention study. Epigenetics 2011 Sep 1;6(9).

2. Scottish Council for Research in Education. The Trend of Scottish Intelligence:A Comparison of the I947 and 1932 Surveys of the Intelligence of Eleven-year-old Pupils. University of London Press. 1949;xxviii(151): 196.

3. Laukkanen MO, Mannermaa S, Hiltunen MO, Aittomäki S, Airenne K, Jänne J, et al. Local hypomethylation in atherosclerosis found in rabbit ec-sod gene. Arterioscler Thromb Vasc Biol 1999 09//;19(9):2171-2178.

4. de Bilbao F, Arsenijevic D, Moll T, Garcia-Gabay I, Vallet P, Langhans W, et al. In vivo over-expression of interleukin-10 increases resistance to focal brain ischemia in mice. J Neurochem 2009 07//;110(1):12-22. 